\title{
Technical note: The role of circulating low-density lipoprotein levels as a phenotypic marker for Holstein cholesterol deficiency in dairy cattle
}

\author{
S. Saleem, C. Heuer, C. Sun, D. Kendall, J. Moreno, and R. Vishwanath ${ }^{1}$ \\ Inguran, LLC dba STGenetics, 22575 SH6 South, Navasota, TX 77868
}

\begin{abstract}
With the recent discovery of a Holstein cholesterol deficiency (HCD) haplotype, the USDA has labeled many dairy animals as HCD carriers based on haplotype and pedigree analysis. We set out to investigate the effect of HCD status on various cholesterol transport molecules, namely low-density lipoprotein cholesterol (LDL-C), high-density lipoprotein cholesterol, and triglycerides in both males and females. A genome-wide association study was also conducted to narrow down the genomic region correlated with varying LDL-C levels. In the study, 34 HCD carrier animals showed significantly lower cholesterol and LDL-C levels compared with their 34 closely related, non-HCD controls. The genome-wide association study based on 73 animals using 56,198 SNP markers revealed an association with chromosome 11 in the region of $66,218,925$ to $66,946,746 \mathrm{bp}$. We also tested the effect of HCD status on sperm quality traits using fresh ejaculates and frozen-thawed semen samples, but did not find any discriminating effects. Our study has demonstrated the use of LDL-C as a key phenotypic marker for determining HCD status in dairy cattle and this is the first study that clearly shows a cause-effect relationship of the HCD haplotype on circulating LDL-C.
\end{abstract}

Key words: Holstein cholesterol deficiency, cholesterol, Holstein, low-density lipoprotein-cholesterol, sperm quality, dairy

\section{Technical Note}

In the genomics era, scientists and breeders are constantly searching for recessive alleles associated with phenotypes of interest. Identification of new phenotypes and their genetic components allow farmers to make informed decisions about their herds and raise healthy and productive animals. Many other recessive haplotypes have been discovered and a simple genomic

Received December 22, 2015.

Accepted March 14, 2016.

${ }^{1}$ Corresponding author: vish@stgen.com test can reveal carriers versus noncarriers; for example, bovine leucocyte adhesion deficiency, JH1 haplotype in Jerseys (Sonstegard et al., 2013), and the JH2 haplotype (VanRaden et al., 2014). A recent report by Kipp et al. (2015) highlighted the presence of the Holstein cholesterol deficiency (HCD) haplotype, which affects $8.7 \%$ of the Holstein population in Germany alone. The key phenotypic traits associated with the HCD haplotype are nontreatable lethal diarrhea and insufficient development resulting in death within the first few months after birth (Kipp et al., 2015). Key findings also included decreasing levels of circulating cholesterol in heterozygous animals and low to no cholesterol in homozygous animals. Since their initial report, the USDA has officially assigned many animals with HCD carrier status based on haplotype tests and pedigree analysis (VanRaden and Null 2015). As a result, many animals at STGenetics (Navasota, TX) were also assigned the HCD carrier status. STGenetics is a US-based genetics company engaged in the development of dairy cattle genetics for sale of semen and embryos both domestically and internationally. The objective of the current study was to investigate if there was any association of the assigned HCD status of these animals to a phenotypic trait. Specifically, we set out to determine (1) if HCD status had an effect on circulating cholesterols, lowdensity lipoprotein cholesterol (LDL-C), high-density lipoprotein cholesterol (HDL-C), and triglycerides, (2) if a correlation existed between LDL-C levels independent of HCD status and a chromosomal location, and (3) if sperm quality features of fresh and frozen-thawed semen samples were affected by the HCD status of the male animals.

Primary analysis by Kipp et al. (2015) suggested lower levels of cholesterol in carriers for the HCD haplotype. Using haplotype and pedigree analysis, the USDA assigned several STGenetics animals in to different categories based on HCD status. A nominal assignment of the carrier status is HCD0 as a confirmed noncarrier, HCD1 as a confirmed carrier, HCD2 as a lethal homozygous, and HCD3 as an indeterminate carrier. We have used the information on HCD carrier status provided by the USDA to determine other phenotypic 
characteristics of the HCD carrier haplotype. To ascertain if animals of HCD1 status had specific phenotypic traits, males and females were tested for varying levels of cholesterol, and closely related HDL-C, LDL-C, and total triglycerides. Controls were selected as sibs of HCD1 animals with USDA HCD0 designation. Peripheral blood from a venipuncture was collected in serum separator tubes and centrifuged within an hour of collection. Cholesterol, HDL-C, LDL-C, and triglyceride levels were measured directly in serum samples at Marshfield Labs using commercial kits manufactured by Beckman Coulter Inc. (Brea, CA; personal communication with Frances Moore, Marshfield Labs, Veterinary Services, Marshfield, WI). Briefly, total cholesterol was measured through a series of enzymatic reactions. Cholesterol esters in the samples were hydrolyzed using a reagent containing cholesterol esterase to release free cholesterol, followed with oxidation by cholesterol oxidase to produce hydrogen peroxide, which further reacted with 4-aminoantipyrine and phenol, in the presence of peroxidase, to produce a red-colored complex. This complex was measured spectrophotometrically at $540 / 600 \mathrm{~nm}$, with the increase in absorbance being directly proportional to the total cholesterol concentration in the sample. Similarly, triglycerides were assayed through a series of coupled enzymatic reactions. Triglycerides in the samples were hydrolyzed using lipases to produce glycerol. The glycerol was then phosphorylated to produce glycerol-3-phosphate, which was further oxidized by glycerol phosphate oxidase to produce hydrogen peroxide. The formed hydrogen peroxide reacted with 4 -aminophenazone and $\mathrm{N}, \mathrm{N}$-bis(4sulfobutyl)-3,5-dimethylaniline disodium salt, in the presence of peroxidase, to produce a blue-colored complex. This complex was measured spectrophotometrically at $660 / 800 \mathrm{~nm}$, with the increase in absorbance being directly proportional to the triglyceride concen- tration in the sample. The LDL-C in the samples was separated from the other lipid fractions and selectively measured using a 2-step enzymatic process. In the first step, a unique detergent was used to solubilize cholesterol from non-LDL lipoprotein particles, and this cholesterol enzymatically consumed by cholesterol esterase, cholesterol oxidase, peroxidase, and 4-aminoantipyrine to generate a colorless end product. In the second step, a different detergent was added to solubilize cholesterol specifically from the LDL, followed by an enzymatic reaction with cholesterol esterase, cholesterol oxidase, and a chromogen system to yield a blue-colored complex. This colored complex was measured bichromatically at 540/660 nm, with the increase in absorbance being directly proportional to the LDL-C concentration in the sample. A similar 2-step detergent solubilization and enzymatic reaction was used to measure HDL-C in our samples.

Table 1 describes the sample size, sex, and age of the selected animals; females used to test the metabolites were either virgin or pregnant heifers and were not lactating. Blood samples were collected from bulls in active semen production in August 2015 on a commercial dairy in Fond du Lac, Wisconsin, and from virgin and pregnant heifers at a commercial dairy farm in South Charleston, Ohio, in October 2015 (Table 1). To identify a causal mutation or genomic region associated with the low LDL-C levels, a genome-wide association study (GWAS) was performed on 73 animals (31 females and 42 males; Table 1). Animals were genotyped using a collection of the Illumina Bovine BeadChip (Illumina Inc., San Diego, CA) and GeneSeek Bovine BeadChip (GeneSeek, Lansing, MI) comprising 56,198 total SNP. The GWAS was conducted using a sliding window multiple regression of 15 markers at a time. Test statistics were computed based on likelihood ratio tests against the null model that only included an in-

Table 1. Sample size and age of the selected animals belonging to the different Holstein cholesterol deficiency (HCD) groups and to carry out the genome-wide association study (GWAS)

\begin{tabular}{llccc}
\hline Item & $\begin{array}{l}\text { HCD } \\
\text { Status }\end{array}$ & $\begin{array}{c}\text { Sample } \\
\text { size }\end{array}$ & $\begin{array}{c}\text { Average } \\
\text { age (mo) }\end{array}$ & $\begin{array}{c}\text { Median } \\
\text { age (mo) }\end{array}$ \\
\hline $\begin{array}{l}\text { HCD groups } \\
\text { Males }\end{array}$ & HCD 1 & 19 & 28.6 & 25.9 \\
& HCD 3 & 3 & 19.7 & 19.1 \\
Females & Control & 22 & 28.4 & 23.5 \\
& HCD 1 & 15 & 11 & 12.1 \\
GWAS & HCD 3 & 16 & 15.2 & 17 \\
Males & Control & 12 & 14.6 & 26 \\
& & & & 19 \\
Females & HCD 1 & 19 & 28.73684 & 25 \\
& HCD 3 & 3 & 19.66667 & 12 \\
& Control & 20 & 28.84211 & 19 \\
\hline
\end{tabular}


tercept and sex as fixed effects. An ANOVA was carried out to test for the effect of HCD status of the animals on the measured phenotypes (Figure 1).
Both cholesterol and LDL-C levels were significantly reduced in HCD1 males and females relative to their controls (cholesterol: males HCD1 $=47.47 \pm 3.19 \mathrm{mg} /$

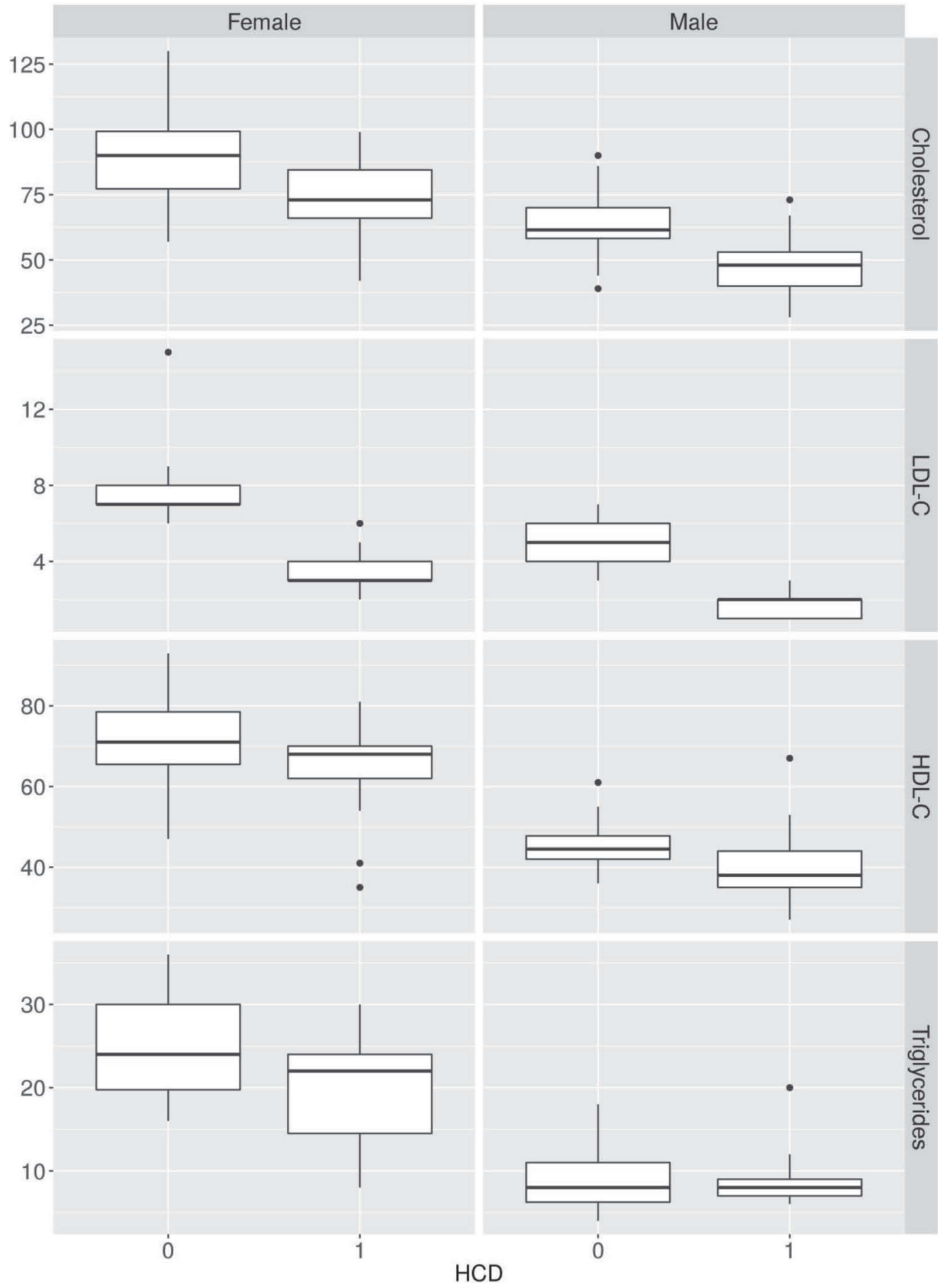

Figure 1. Boxplots of the analyzed phenotypes across males and females and animals classified as Holstein cholesterol deficiency (HCD) 0 and HCD1. Boxes represent the range between first and third quartile (IQR) with the inner horizontal line being the median. Whiskers extend to at most 1.5 times of the IQR, whereas the outliers (dots) exceed this threshold. LDL-C = low-density lipoprotein cholesterol; HDL-C $=$ highdensity lipoprotein cholesterol. 
$\mathrm{dL}$, males HCD0 $=63.86 \pm 2.96 \mathrm{mg} / \mathrm{dL}$; females HCD1 $=71.6 \pm 3.59 \mathrm{mg} / \mathrm{dL}$, females $\mathrm{HCD0}=89.5 \pm 4.01$ $\mathrm{mg} / \mathrm{dL}$; LDL-C: males HCD1 $=1.89 \pm 0.35 \mathrm{mg} / \mathrm{dL}$, males $\mathrm{HCD} 0=4.72 \pm 0.32 \mathrm{mg} / \mathrm{dL}$; females $\mathrm{HCD} 1=$ $3.53 \pm 0.39 \mathrm{mg} / \mathrm{dL}$, females HCD0 $=7.91 \pm 0.44 \mathrm{mg} /$ $\mathrm{dL} ; P \leq 0.0001 ;$ Figure 1). We also tested cholesterol and LDL-C levels of a single homozygous (HCD2) calf whose blood cholesterol levels were markedly reduced and also showed signs of episodic fever and poor development (cholesterol: $<10 \mathrm{mg} / \mathrm{dL}$, LDL-C: $<1 \mathrm{mg} / \mathrm{dL}$ ). Based on the GWAS, the window with the strongest association ( $-\log _{10} P$-value of 6.067$)$ was located on chromosome 11 in the region of 66218925 to 66946746 bp (Figure 2), which is in proximity of the HCD haplotype location reported by Kipp et al. (2015).

Presence of altered levels of cholesterol-to-phospholipid ratios in certain species reduce sperm susceptibility to cold shock (Watson, 1981; Crockett, 1998). Cholesterol helps stabilize membranes and also mediates the movement of molecules across the cells. Sperm quality also suffers due to cryopreservation because of this altered cholesterol-to-phospholipid ratio. Cryopreservation is important as this process greatly facilitates extended sperm storage, sorting, and distribution. In general, freeze-thawing damages the sperm, which can further reduce sperm viability, motility, acrosomal integrity, and fertilization capacity (Holt, 2000). Damage to sperm can be prevented by changing and modifying the external environment, such as cryodiluent medium, rate of freezing, and thawing (Vishwanath and Shannon, 2000). However, specific attributes of the sperms' internal environment can also be tested for improved sperm quality post-thawing. Phospholipids and cholesterol in the sperm membranes help maintain structural integ-

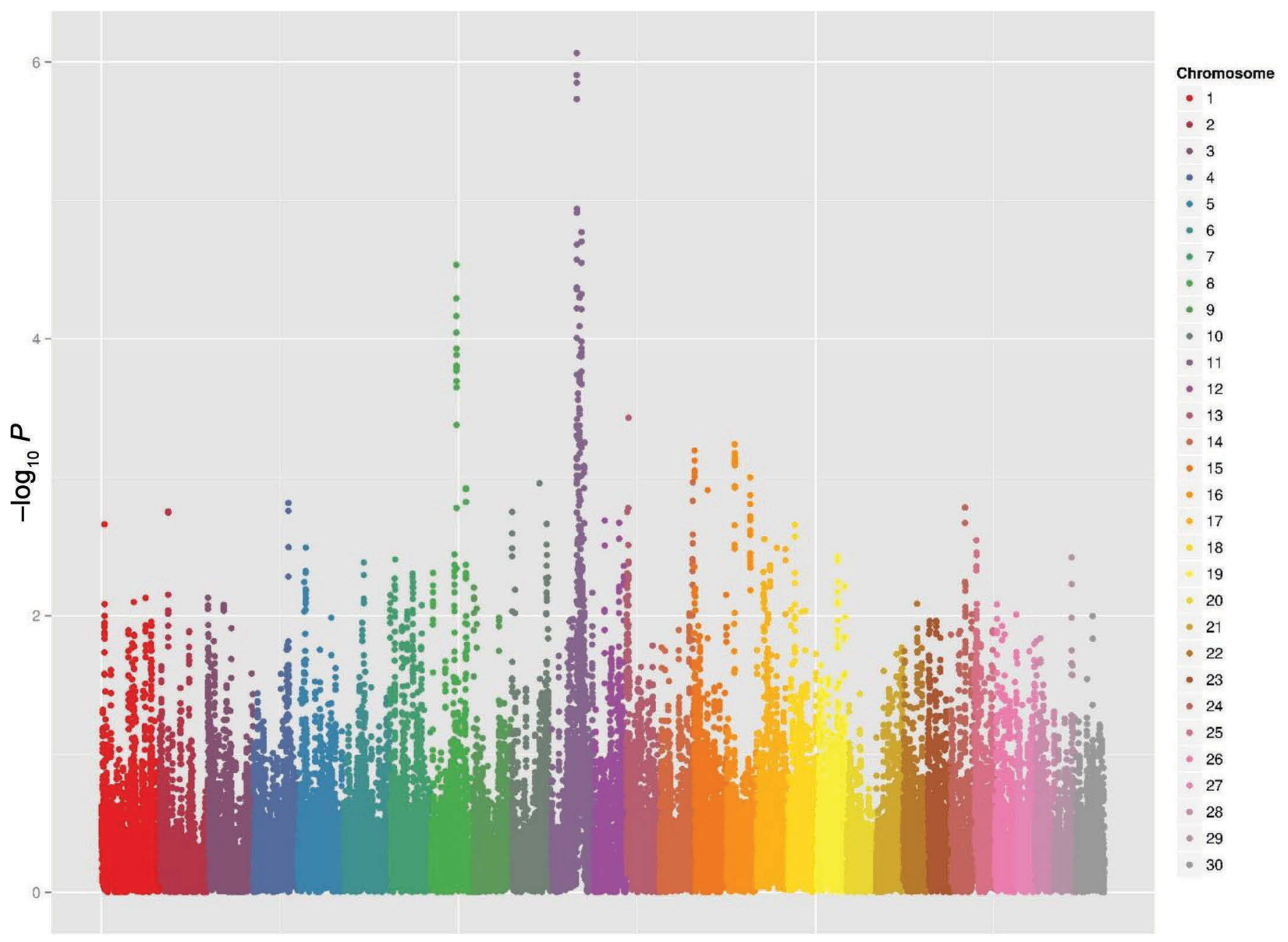

Figure 2. Sliding window genome-wide association study for low-density lipoprotein C. In total 73 male and female animals across all Holstein cholesterol deficiency (HCD) classes with 56,198 SNP genotypes were used in windows of 15. 
Table 2. Sample size of males used to measure association between Holstein cholesterol deficiency (HCD) status and sperm traits, and comparison of sperm quality traits between HCD carrier and control animals

\begin{tabular}{|c|c|c|c|c|c|c|c|}
\hline Item $^{1}$ & $\begin{array}{l}\text { Sample } \\
\text { size }\end{array}$ & $\begin{array}{l}\text { Average } \\
\text { age (mo) }\end{array}$ & $\begin{array}{l}\text { Median } \\
\text { age (mo) }\end{array}$ & Groups & Estimate & SE & $\operatorname{Pr}(>|z|)$ \\
\hline \multicolumn{8}{|l|}{ HCD Status } \\
\hline Control & 1,836 & 57.8 & 57.4 & & & & \\
\hline \multicolumn{8}{|l|}{ Trait } \\
\hline Ejaculate volume & & & & $1-0$ & 0.27 & 0.24 & 0.50 \\
\hline \multirow{3}{*}{$\begin{array}{l}\text { Ejaculate motility } \\
(\%)\end{array}$} & & & & $1-0$ & -1.09 & 0.76 & 0.31 \\
\hline & & & & $3-0$ & -1.65 & 1.41 & 0.46 \\
\hline & & & & $3-1$ & -0.56 & 1.58 & 0.93 \\
\hline \multirow[t]{3}{*}{ 0-h motility (\%) } & & & & $1-0$ & -0.80 & 0.45 & 0.16 \\
\hline & & & & $3-0$ & 0.55 & 0.88 & 0.80 \\
\hline & & & & $3-1$ & 1.35 & 0.98 & 0.33 \\
\hline 3-h motility (\%) & & & & $1-0$ & -0.44 & 0.65 & 0.77 \\
\hline
\end{tabular}

${ }^{1}$ Ejaculate volume, ejaculate motility immediately postejaculation, and 0 - and 3-h motility and acrosome quality were measured after thawing. Multiple contrast tests (comparisons of means) were conducted to determine significant differences among the different HCD groups HCD0 (0), HCD1 (1) and HCD3 (3).

rity at room temperature and at very low temperatures. As HCD carrier animals show low levels of cholesterol and LDL-C, we investigated the effect of HCD status on various sperm characteristics in Holstein males. To determine the relationship between cholesterol deficiency and semen collection attributes, we queried the STGenetics semen collection database, where measures of fresh ejaculate volume, initial motility, and postthaw motility of HCD0 (334), HCD1 (18), and HCD3 (7) males were recorded (Table 2). Fresh ejaculate volume was measured using a volumetric or gravimetric method. For motility, a $10-\mu \mathrm{L}$ ejaculate sample was diluted with $500 \mu \mathrm{L}$ of Tyrode's lactate pyruvate buffer $\left(\mathrm{pH} 7.3\right.$; Bavister and Yanagimachi, 1977) at $36^{\circ} \mathrm{C}$, and evaluated on a slide under phase contrast conditions using a Nikon Eclipse 80i microscope (Nikon, Melville, NY). Post-thaw motility at 0 and $3 \mathrm{~h}$ were measured by thawing frozen semen samples at $38^{\circ} \mathrm{C}$ for $30 \mathrm{~s}$, and then evaluated immediately on a warm slide at $37^{\circ} \mathrm{C}$. This observation was repeated again after a 3 -h incubation of the thawed sample at $34.5^{\circ} \mathrm{C}$. Acrosome status was determined by counting intact acrosomes between 0 and $3 \mathrm{~h}$ post-thawing at $40 \times$ magnification using phase contrast microscopy. In total 28,215 observations were used in a mixed model with heard-year-season, age in months, and HCD status as fixed effects and a random sire effect (permanent environment). No effect of HCD status was observed on fresh ejaculates and post-thaw semen quality attributes (Table 2). Recent work by J.
B. Cole (Animal Genomics and Improvement Laboratory, Agricultural Research Service, USDA, Beltsville, $\mathrm{MD}$, personal communication) has indicated no effect of HCD heterozygosity on milk production.

Our findings suggest that levels of circulating LDL-C are indicative of an animal's HCD status. Menzi et al. (2016) recently identified the causal mutation leading to HCD. The mutation was localized to the bovine apoB gene $(A P O B)$, which encodes for apolipoprotein $\mathrm{B}$, a main component of chylomicrons and LDL-C. It exists as either apoB-48 or apoB-100 in plasma, with apoB-48 being synthesized in the gut whereas apoB100 is synthesized in the liver, with help of a unique posttranscriptional editing process. The LDL encapsulate and transport cholesterol in the blood stream and deliver it to tissues and cells for maintenance of membranes, regulation of cell signaling pathways, and fat storage (Frostegård et al., 1990; Hisamatsu-Sakamoto et al., 2008). In addition to regulatory roles, apoB-100 in vesicle membranes also serve as a ligand for LDLreceptor-mediated uptake by cells. Apolipoprotein B is the sole protein component of LDL molecules, which also consist of hydrophobic molecules such as triglycerides and cholesteryl esters (Dominiczak and Muriel, 2011; reviewed in Whitfield et al., 2004). A similar condition, called familial hypobetalipoproteinemia (FHBL), is also found in humans. It is marked by low levels of cholesterol and LDL-C, resulting in the body's inability to absorb and transport fats (Whitfield et 
al., 2004). A majority of the cases of FHBL have been linked to mutations in the $A P O B$ gene, which result in the formation of a truncated apoB protein of various sizes (Schonfeld, 2003). Many individuals with FHBL develop abnormal deposition of fat in the liver producing a fatty liver. However, in our study, the HCD2 calf did not show signs of a fatty liver on closer examination (data not shown), but did suffer from constant fever and inability to gain weight despite maintaining a healthy appetite. Humans with FHBL demonstrate a variety of phenotypes ranging from failure to thrive to neurological complications and different levels of hepatic disease. Early diagnosis and treatment are key in ensuring and prolonging a healthy life (Schonfeld, 2003).

Interestingly, triglyceride levels also showed a decreasing but nonsignificant trend in HCD1 animals compared with their controls; this is not surprising, as triglycerides form the basic unit of many lipids and LDL-C. Based on our findings, the highly significant differences in cholesterol and LDL-C levels between HCD carriers and controls support the discriminative power of circulating LDL-C as a biomarker for cholesterol deficiency. The GWAS for LDL-C further showed a strong association with chromosome 11 in proximity of the HCD haplotype. More work is required to determine the nature of the $A P O B$ mutation in Holstein, and our findings encourage further research on the genetic connection between LDL-C and traits of commercial interest using bigger cohorts of animals across many diverse environments.

\section{ACKNOWLEDGMENTS}

We thank all the field staff of STGenetics and acknowledge the use of a large semen evaluation database to extract semen quality information of all suspected HCD animals. We thank Karen Grube and Kerry Balthazar (ST Genetics, Vienna, WI) for assisting with blood collections. We thank Frances Moore, Marshfield Labs, Veterinary Services (Marshfield, WI) for providing additional information on the role of LDL in cattle.

\section{REFERENCES}

Bavister, B. D., and R. Yanagimachi. 1977. The effects of sperm extracts and energy sources on the motility and acrosome reaction of hamster sperm in vitro. Biol. Reprod. 16:228-237.

Crockett, E. L. 1998. Cholesterol function in plasma membranes from ectotherms: Membrane-specific roles in adaptation to temperature. Am. Zool. 38:291-304.

Dominiczak, M. H., and J. C. Muriel. 2011. Apolipoproteins: Metabolic role and clinical biochemistry applications. Ann. Clin. Biochem. 48:498-515.

Frostegård, J., A. Hamsten, M. Gidlund, and J. Nilsson. 1990. Low density lipoprotein-induced growth of U937 cells: A novel method to determine the receptor binding of low density lipoprotein. J. Lipid Res. 31:37-44.

Hisamatsu-Sakamoto, M., N. Sakamoto, and A. S. Rosenberg. 2008. Embryonic stem cells cultured in serum-free medium acquire bovine apolipoprotein B-100 from feeder cell layers and serum replacement medium. Stem Cells 26:72-78.

Holt, W. V. 2000. Fundamental aspects of sperm cryobiology: the importance of species and individual differences. Theriogenology 53:47-58.

Kipp, S., D. Segelke, S. Schierenbeck, F. Reinhardt, R. Reents, C. Wurmser, H. Pausch, R. Fries, G. Thaller, J. Tetens, J. Pott, M. Piechotta, and W. Grunberg. 2015. A new Holstein haplotype affecting calf survival. Interbull Bull. 49:49-53.

Menzi, F., N. Besuchat-Schmutz, N. Fragniere, S. Hofstetter, V. Jagannathan, T. Mock, A. Raemy, E. Studer, K. Mehinagic, N. Regenscheit, M. Meylan, F. Schmitz-Hsu, and C. Drogemuller. 2016. A transposable element insertion in the APOB causes cholesterol deficiency in Holstein cattle. Anim. Genet. 47:253-257. http:// dx.doi.org/10.1111/age.12410

Schonfeld, G. 2003. Familial hypobetalipoproteinemia: A review. J. Lipid Res. 44:878-883.

Sonstegard, T. S., J. B. Cole, P. M. VanRaden, C. P. Van Tassell, D. J. Null, S. G. Schroeder, D. Bickhart, and M. C. McClure 2013. Identification of a nonsense mutation in CWC15 associated with decreased reproductive efficiency in Jersey cattle. PLoS ONE 8:e54872.

VanRaden, P., and D. Null. 2015. Holstein haplotype for cholestero deficiency (HCD). Accessed Jan. 17, 2016. https://www.cdcb.us/ reference/changes/HCD_inheritance.pdf.

VanRaden, P., D. Null, J. Hutchison, D. Bickhart, and S. Schroeder. 2014. Jersey haplotype 2 (JH2). Changes to evaluation system (August 2014). Accessed Jan. 17, 2016. https://www.cdcb.us/ reference/changes/eval1408.htm.

Vishwanath, R., and P. Shannon. 2000. Preservation of bovine semen in the liquid and frozen state. Anim. Reprod. Sci. 62:23-53.

Watson, P. F. 1981. The effects of cold shock on sperm cell membranes. Pages 189-218 in Effects of Low Temperatures on Biological Membranes. G. J. Morris and A. Clark, ed. Academic Press, London, UK.

Whitfield, A. J., P. H. Barrett, F. M. van Bockxmeer, and J. R. Burnett. 2004. Lipid disorders and mutations in the APOB gene. Clin. Chem. 50:1725-1732. 